\title{
Detection for Two-Dimensional Magnetic Recording Systems
}

\author{
Seyed Mehrdad Khatami and Bane Vasić \\ Department of Electrical and Computer Engineering \\ University of Arizona \\ Tucson, AZ, 85721, USA \\ Email: \{khatami,vasic\}@ece.arizona.edu
}

\begin{abstract}
Two dimensional magnetic recording (TDMR) is a novel scheme which envisions reaching $10 \mathrm{~Tb} / \mathbf{i n}^{2}$ density in magnetic recording systems. The feasibility of this density relies largely on sophisticated two-dimensional (2-D) signal processing algorithms. This paper gives a survey on TDMR channel models and 2-D detectors. Our discussion on channel models places an special emphasis on the suitability of the read channel models for the purpose of detector design. Furthermore, a comprehensive review on 2-D detection is given focusing on compatibility of the detectors for the TDMR channel models.
\end{abstract}

Index Terms - Two dimensional magnetic recording (TDMR), read channel model, two-dimensional detectors, Inter-symbol interference (ISI), Data dependent noise

\section{INTRODUCTION}

$\mathbf{M}$ agnetic recording systems have reached a point where the grain size can no longer be reduced due to energy stability constraints. A number of new technologies have been proposed to increase the storage density via other avenues. The most promising technologies include heat-assisted magnetic recording (HAMR) [1], microwave assisted magnetic recording (MAMR) [2], bit-patterned media (BPM) [3] and two-dimensional magnetic recording (TDMR) [4]. Each of these are at different stages of development and pose various unique challenges. HAMR and MAMR rely on a novel $\mathrm{read} /$ write head mechanism, while BPM relies primarily on novel methods for producing highly ordered media. Although an important component, signal processing may not be a deciding factor in determining the feasibility of such systems. TDMR is advantageous because it uses conventional media, relying instead on sophisticated signal processing and coding algorithms, a much less expensive alternative to radically altering the media or the read/write head as required for the other technologies.

Theoretically, TDMR can achieve areal-densities of the order of $10 \mathrm{~Tb} / \mathrm{in}^{2}$ when one bit of information is recorded in one or a few grains. However, formidable challenges need to be addressed before some conclusions on achievable densities can be derived. The nature of write and read processes in TDMR necessitates that the information storage be viewed as a two-dimensional (2-D) system. Therefore, there is a need for developing 2-D signal processing algorithms. One of the major challenges in TDMR signal processing is the detector design. The detector-design paradigm for TDMR is significantly different from approaches used in the literature for two reasons. Firstly, there is severe interference in both down-track and cross-track directions. Hence, it is desirable to develop 2-D detectors in lieu of multi-track detection schemes [5], [6]. Secondly, in contrast to conventional magnetic media, the primary source of noise in TDMR arises from irregularities in the recording medium. These differences also make the detector design difficult. This survey considers the application of 2-D detectors for TDMR channels with data dependent noise. Our main focus is on two 2-D sub-optimal detectors that are applied to the TDMR system and to show how using the data dependent noise information helps to improve the performance.

Since random grain positions and boundaries form the primary source of noise during readback, the detector design also necessitates the development of appropriate TDMR read channel models. TDMR channel models have been studied in [7]. The simplest TDMR channel model is the binary error and erasure channel (BEEC). It models the TDMR channel as a channel where bits are either erased or flipped with assigned erasure and flipping probabilities. Although simple, this model lacks the accuracy needed for detector design. In [8], Kavcic et al. introduced a simple magnetic grain media model called "granular tiling model". In this model, the recoding medium is assumed to consist of randomly shaped tiles covering the medium plane where each tile represent a grain. Using the model in [8], bounds for the capacities of TDMR channels are derived. However granular tiling model is not well suited for the purpose of detector design. In this paper, our main focus is on the Voronoi model [7] and the 2-D Microcell model [9]. In the Voronoi model, the recording medium is modeled as a Voronoi tiling of a plane with every Voronoi region representing a grain. The Voronoi model captures the characteristics of the media noise generally observed in TDMR. The 2-D Microcell model is the generalization of the traditional microtrack model [10] to two dimensions so that each bit is composed of a small number of horizontal and vertical tracks. The 2-D Microcell model is an accurate model for densities which the bit to grain size ratio is on the order of $5-10$. This model enables the analytical derivation of the pattern dependent distributions of the read-head output samples. These models are relatively simple yet accurate which can capture the properties of the media noise and well suited for detector designs.

The one-dimensional (1-D) Maximum a-posterori (MAP) 
detection is theoretically optimal for 1-D inter-symbol interfernce (ISI) channels. However, unlike 1-D MAP detection, 2-D MAP is NP-complete. Therefore, most of 2-D detection techniques are the extended version of 1-D detectors. Various approaches are considered for 2-D detection. The first category of 2-D detection are schemes that utilize BCJR detectors [11] on multiple tracks which is called "Multi-Track" detectors [12]. The core of these techniques is iterative algorithms that operate on multi-track detectors in both directions. However, when the locally optimal multi-track detectors are combined, the global detection becomes sub-optimal. An outline of these algorithms is provided in Section III. The second category is the 2-D detectors which perform sub-optimal decoding on the full graph. The Generalized Belief Propagation (GBP [13]), which originates from statistical physics, is in the second category of detectors and has been first considered in the context of 2-D detection by Shental et al. [14]. The GBP detector operates on an undirected graphical model of a twodimensional Gaussian channel with memory. It infers the symbol a posteriori probabilities (APPs) using a message passing algorithm which takes into account the existence of loops in a graph. Using GBP is a natural way of exploiting the 2-D characteristics of ISI, and it was shown in [14] that the performance of GBP is almost the same as the MAP detector.

In case of TDMR read channel model, the read-head output samples are also contaminated by the data dependent media noise. Therefore the detector schemes have to be modified to incorporate with data dependent noise model.

The paper is organized as follows: In Section II, the readchannel models are reviewed and the Voronoi and Microcell model are explained in this section. Section III outlines the detection problem and reviews most of the important 2-D detectors. Finally, Section IV concludes the paper.

\section{CHANNEL MODEL}

In this section we describe TDMR channel models which can easily applied in design of near-optimum detectors. The $\mathrm{read} /$ write channel model for TDMR consists of three components: the data writing process, 2-D channel and the readback process. The write process ,the TDMR channel and the readback process are introduced in Sections II-A, II-B and II-C, respectively.

\section{A. Writing Process}

The approach to data storage in TDMR is significantly different from those of today's recording systems. Firstly, TDMR attempts to store one bit in very few grains of conventional magnetic media, the ultimate goal being that of storing 1 bit/grain (today's systems use about 20 grains per bit). To ensure a sufficiently large magnetization field, the head is made larger than the track width. This relaxes head design constraints, but in order to achieve very narrow tracks, each sweep of the write head partially overlaps with the previous track. This technique is known as shingled writing [4].

Such an aggressive write process, along with the lack of ordering of grains as in the conventional media, may cause some bits to be over-written and some to not be written at all.

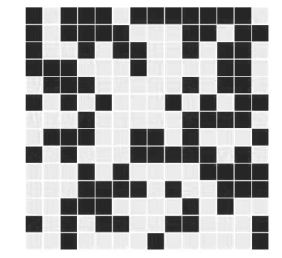

(a)

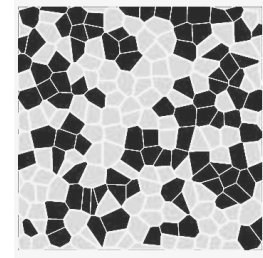

(b)

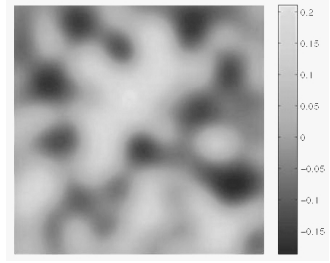

(c)
Fig. 1: Write/read model for the Voronoi medium: (a) Desired magnetization of an ideal medium. (b) Magnetization of a non-ideal medium. (c) Readback signal (before sampling). We assume the readback impulse response to be a truncated 2D Gaussian pulse of unit energy with half-maximum of 1 bitperiod and a span of 3 bit-periods in both dimensions.

We note that bits lost to write-errors can never be recovered by any detector. We can only hope to recover such information by the use of constrained and error-correcting codes. This is an unavoidable price of making the dimension of bits so small.

\section{B. TDMR Channel Models}

One of the critical challenges in TDMR is the channel modeling for detector design. In contrast to conventional recording systems, the primary source of noise comes from random grain positions and the lack of knowledge of these boundaries during the readback process. The TDMR models discussed here, are the Voronoi and the 2-D Microcell model.

1) Voronoi Model [7]: In this model, the recording medium can be visualized as the Voronoi tiling of the shifted graincenters with their regions representing the grains. The randomness in the shape and position of grains is modeled by shifting the grain-centers randomly. Each region represents a set of all points in the media closer to the selected grain center. The shift of the grain-centers from their ideal positions is a random variable (R.V.) of a known probability distribution in a manner that the shifted grain-centers are always within their corresponding cells.

The write-head simply assumes that the medium is ideal and attempts to write at the center of each cell in a raster fashion. The grain whose center is within the cell is then appropriately magnetized. Fig. 1 shows writing/reading process on a square lattice. It illustrates the difference between the magnetization of an ideal medium and an actual medium as a result of the writing process. Figures 1(a) and 1(b) show the magnetization of an ideal and the actual medium, respectively. The grains with magnetization $+1 /-1$ are colored light and dark, respectively.

2) 2-D Microcell Model [9]: In this model, the grains which straddle the cell boundaries are modeled by small tracks displaced from the ideal cell borders. In the Microcell model, the cell on which the signal is written, is divided into $N$ equally sized smaller tracks called microtracks which are oriented in vertical and horizontal directions. The perturbations are modeled as the random position of microtrack boundaries. Thus, the irregularities of the non-ideal cells are modeled by microtracks displaced from the ideal position. A non-ideal cell with displaced transition boundaries is call a "microcell". The 


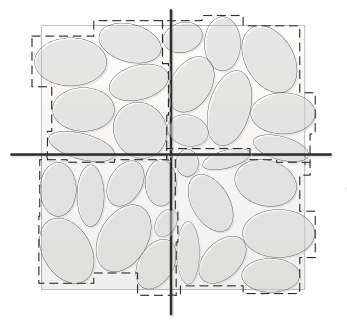

(a)

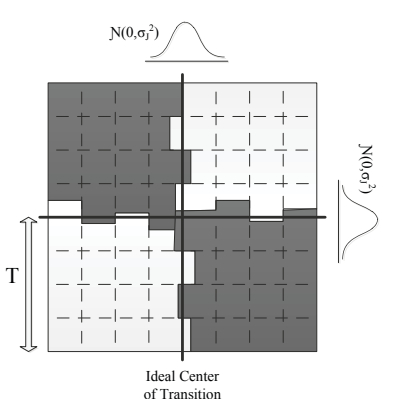

(b)
Fig. 2: (a) A realization of the distribution of grains in the Microcell model. (b) The 2-D Microcell model for $2 \times 2$ cell

transition boundaries represented by a number of microtracks are independently and randomly displaced from the desired (ideal) transition position. Fig 2(a) shows the Microcell model as a simple and natural way to approximate the irregular boundaries of the medium. Fig. 2(b) illustrates the differences between ideal cells and microcells in the microcell model. $1 /-1$ bits are colored as light/dark, respectively. Each shift is chosen from a distribution which is typically considered to be a truncated Gaussian distribution (see Fig. 2(b)). The Microcell model can be considered as a 2-D generalization of the microtrack model [10].

\section{Readback Process}

During readback, the read-head picks up magnetization from multiple bits in the down-track and cross-track directions. Hence, there is severe inter-symbol interference (ISI) in both directions. The readback signal is obtained by convolving the magnetization of the medium with the read-head response and then sampling at cell-centers.

In the remainder of this section, the readback process is formulated to determine the properties of the output samples based on the discussions in [9]. To facilitate the discussions, we introduce the concepts of an ideal medium, an ideal and non-ideal cell. These concepts are applicable to both Voronoi and Microcell models. A medium is "ideal" if the bit areas are equally sized and regularly spaced squares. We refer to the bit areas in an ideal medium as "ideal cells". In our channel model, all instances of recording medium are assumed to be produced by independent perturbation of the ideal medium. We refer to the perturbed bit areas as "non-ideal cells". The irregularities of the non-ideal cell are estimated as a random process of perturbing the ideal cell.

Let $x_{i, j} \in\{-1,+1\}$ be the input data bits and let $x_{c}\left(t_{1}, t_{2}\right)$ be the continuous magnetization of the recording medium after the write process at position $\left(t_{1}, t_{2}\right)$. Given $x_{i, j}$, variation of $x_{c}\left(t_{1}, t_{2}\right)$ is due to the different realizations of the non-ideal cells. Fig. 1(b) and Fig. 3(b) show $x_{c}\left(t_{1}, t_{2}\right)$ for the Voronoi and 2-D Microcell model, respectively. The readback signal samples are obtained by convolving the magnetization of the recording medium with the 2-D read-head response and then sampling the resulting signal at cell centers $\left(i T+\frac{T}{2}, j T+\right.$ $\frac{T}{2}$ ) where $i, j$ are integers. The response of the read-head,

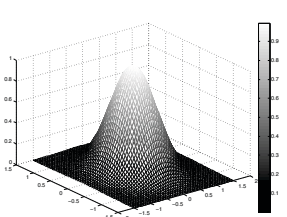

(a)

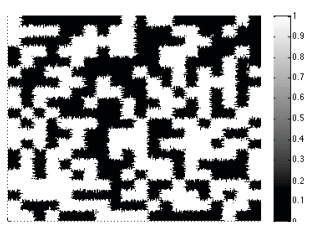

(b)

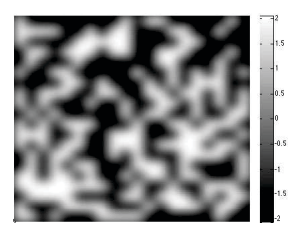

(c)
Fig. 3: Visualization of read channel model. a) Read-head impulse response. b) Input signal for Microcell model. c) Output signal after convolving the read-head.

$h\left(t_{1}, t_{2}\right)$, can be obtained by using the method proposed in [15]. In this paper, we assume the read-head response to be a truncated 2-D Gaussian pulse which spans $3 \times 3$ cells. We choose a $3 \times 3$ for the read-head response since it is reasonably small yet sufficient to consider the ISI effect. Moreover, it is also the typical size considered in many pervious works ( [7], [14]).

Let $y_{i, j} \in \mathbb{R}$ be the noiseless readback signal samples and $r_{i, j} \in \mathbb{R}$ be the noisy readback signal samples. Due to major improvements in magnetic recording readers, the noise is mostly composed of media noise with small portion of electric noise. The noise free readback signal samples can be expressed as the discrete convolution of the input data bits $\left(x_{i, j}\right)$ and the discretized read-head response $\left(h_{k_{1}, k_{2}}\right)$ :

$$
y_{i, j}=\sum_{k_{1}=-1}^{+1} \sum_{k_{2}=-1}^{+1} h_{k_{1}, k_{2}} x_{i-k_{1}, j-k_{2}}
$$

Let $C_{i, j}=\left\{\left(k_{1}, k_{2}\right) \mid i-1 \leq k_{1} \leq i+1, j-1 \leq k_{2} \leq\right.$ $j+1\}$ be the set of local neighborhood indices of bit $(i, j)$ and $\mathbf{x}_{C_{i, j}}=\left\{x_{k_{1}, k_{2}} \mid\left(k_{1}, k_{2}\right) \in C_{i, j}\right\}$ be all the input data bits which contribute to the read-back signal sample $y_{i, j}$.

In the non-ideal case for both channel models, The readhead output samples can be written as follows:

$$
\begin{aligned}
& r_{i, j}=\sum_{k_{1}=-1}^{+1} \sum_{k_{2}=-1}^{+1} \\
& \iint_{A_{i-k_{1}, j-k_{2}}^{\prime}} h\left(i T+\frac{T}{2}-\tau_{1}, j T+\frac{T}{2}-\tau_{2}\right) x_{i-k_{1}, j-k_{2}} d \tau_{1} d \tau_{2}
\end{aligned}
$$

where $A_{k_{1}, k_{2}}^{\prime}$ is the area spanning the $\left(k_{1}, k_{2}\right)^{t h}$ grain (microcell in case of Microcell model). The output samples, $r_{i, j}$, can be obtained by sampling the read-head output signal for the Voronoi and Microcell model in Fig. 1(c) and Fig. 3(c) at the center of cell $(i, j)$. The non-ideal read-back signal sample can also be written as

$$
r_{i, j}=y_{i, j}+n_{i, j}
$$

This change in output for the ideal and non-ideal case is considered as "media noise". $n_{i, j}$ is the media noise component for cell $(i, j)$. This noise depends not only on grain (microcell in case of Microcell Model) shapes in $C_{i, j}$, but also on the polarity of the magnetization as it will be discussed later. As a result, the media noise is a data dependent noise 


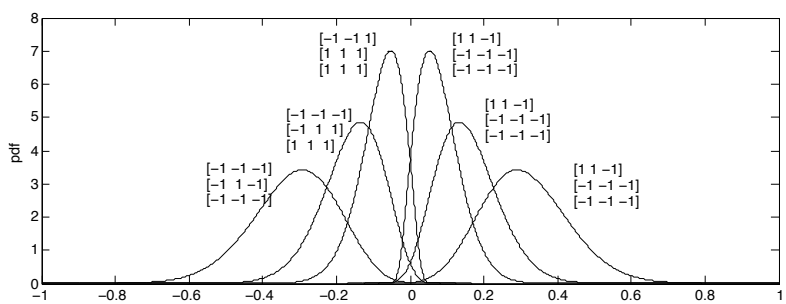

Fig. 4: An example of data dependent media noise distributions for the Microcell model with various input data patterns.

and from (1), its conditional probability density function (pdf) given the local neighborhood data bits can be written as

$$
p\left(r_{i, j} \mid \mathbf{x}_{C_{i, j}}\right)=p\left(r_{i, j}-y_{i, j} \mid \mathbf{x}_{C_{i, j}}\right)=p\left(n_{i, j} \mid \mathbf{x}_{C_{i, j}}\right)
$$

The data dependent noise, $n_{i, j}$, is caused by the difference between the input data for two neighboring bits in $C_{i, j}$. Therefore, media noise components caused by the common sides depend on the value of the corresponding neighboring bits. If two neighboring bits have the same value, there is no media noise caused by their common side. Fig. 4 shows the media noise distributions for the Microcell model for several input data pattern. As it is shown, flipping the input data results in a symmetrical distribution about $y$-axis.

\section{Detection}

The problem of detection in 2-D ISI channels arrises in many applications. The literature is rich with various methods for reducing the effect of 2-D ISI. However, most of 2-D detection techniques are the extended version of 1-D detectors. We partition 2-D detectors into two categories. The first category is the multi-track extensions of 1-D algorithms such as the BCJR and the soft Viterbi algorithm. In [16], Nabavi et al. proposed a 2-D generalized partial response (GPR) equalizer followed by conventional 1-D Viterbi detector for processing signals read from 3 adjacent tracks. A joint-track detection using a 1-D equalizer with a 2-D GPR target was proposed in [17]. The 2-D soft output Viterbi algorithm (SOVA) detection using two 1-D SOVAs for the along and cross-track directions was proposed in [18]. However, because the 2-D SOVA detection is based on a 1-D GPR target, it offers smaller gain than the multi-track detection. In [19], an iterative algorithm to exchange soft information between two soft-input soft-output (SISO) 1-D detectors is implemented. 2-D Decision Feedback Equalizer (DFE) and MAP-DFE hybrid equalization schemes are considered in [20]. In [21], separable 2-D ISI channel is used for transmission. Non-binary column MAP detector concatenated to a binary row MAP detector followed by LDPC decoder is considered. Iterative techniques and coding gain enables [21] to approach the non-ISI curve within $1 \mathrm{~dB}$. In [22] Chen et al. developed an iterative soft-decision feedback zig-zag MAP algorithm for good performance in low SNR. In a recent work [23], Chen et al. developed several turbo feedback based multi-track MAP algorithm which show $2 \mathrm{~dB}$ SNR gain over non-iterative methods.

All these detection schemes are multi-track rather than 2$\mathrm{D}$, and consequently cannot fully exploit the characteristics of the channel detection with 2-D interference. The second category is the full-graph sub-optimal detector such as the Generalized Belief Propagation (GBP [13]). GBP algorithm has been first used for 2-D detection by Shental et al. [14]. The detector operates on an undirected graphical model of a two-dimensional Gaussian channel with memory. It infers the symbol a posteriori probabilities (APPs) using a message passing algorithm which takes into account the existence of loops in a graph.

In this section, we discuss two different 2-D sub-optimal detectors that are applied to the TDMR system. The first one is extended version of 1-D MAP detector for separable 2-D channels and the second one is the GBP detector.

\section{A. Separable 2-D MAP Detector}

In [21], separable 2-D ISI channel is used for transmission. The ISI channel is separable if the 2-D ISI matrix can be written as a product of a column and a row vector. Thus, $H=\bar{U} \bar{V}$ where $\bar{U}$ and $\bar{V}$ are column and row vectors, respectively. It can be shown that if the channel is separable, then the channel can be considered as two 1-D channels which one results in ISI along the row and the other one results in ISI along the column. So an iterative detector is developed accordingly which consists of a 1-D non-binary column MAP detector concatenated to a 1-D binary row MAP detector. In [21], an LDPC decoder is also incorporated which enables this detector to approach the non-ISI curve within $1 \mathrm{~dB}$.

\section{B. The GBP Detector}

The main difference between GBP and BP is that the messages are sent between regions of nodes instead of node to node message passing. As it is shown in Fig. 5(a) for a case of $4 \times 4$ grid, there exists many cycles in 2-D ISI channel factor graph. As a result, the tree-like assumption used in BP does not hold and BP approximation is poor. In GBP algorithm, sets or regions of nodes will send messages to other regions of nodes. GBP uses region graph method to specify regions and messages. Fig. 5(c) shows the regions and the messages between the regions. Yedidia et al explained GBP in details in [13]. The major advantage of GBP is benefiting from region to region message passing instead of node to node message passing.

\section{Compatibility of Detection Methods to TDMR Read Chan- nel Models}

In order to get close to optimal MAP detector, the detector needs to account for the correlation between noise samples. Since the noise samples are dependent to their local neighborhood input, the detector must incorporate the data dependent media noise distributions in calculating a posteriori probabilities. In multi-track methods, the noise distributions must be accounted for in calculating the metrics of the branches. However, the incorporation of the noise distributions is not possible for all the methods. In the GBP detector, by selecting the regions the same as the span of the ISI, data dependent media noise distributions can be applied in calculating local $a$ priori probabilities to improve the performance of the detector. 


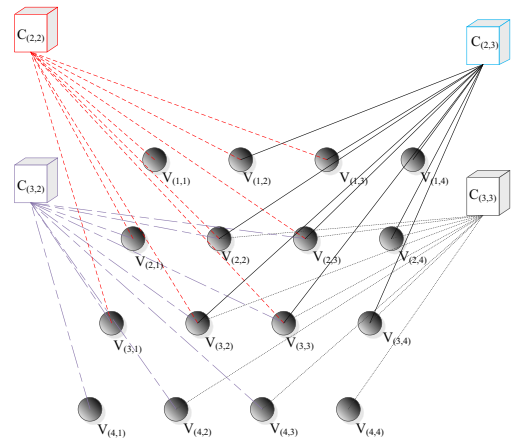

(a)

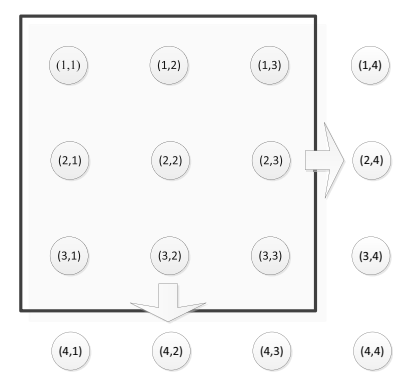

(b)

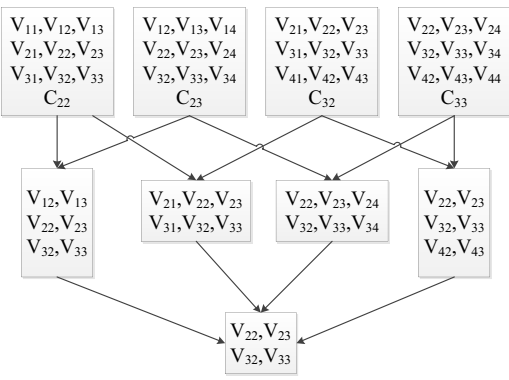

(c)

Fig. 5: a) Factor graph for the $4 \times 4$ 2-D ISI channel. b) Sliding regions include all the nodes contributing to the corresponding factor node. c) A region graph generated using the cluster variation method [13].

\section{CONCLUSiON}

In this paper, we have provided an overview of the read channel models for TDMR. The main focus was on the Voronoi and 2-D Microcell model for the propose of detector design. Moreover, we have reviewed the important works on two-dimensional detection algorithms. Our main goal was to provide a groundwork for addressing the challenges in incorporation of the TDMR read channel models in detector design.

\section{ACKNOWLEDGMENT}

This work is funded by IDEMA ASTC and partially by NSF under Grant CCF-0963726. The authors would like to thank Anantha Raman Krishnan for his helpful suggestions and acknowledge his contribution in development of the Microcell model.

\section{REFERENCES}

[1] R. Rottmayer, S. Batra, D. Buechel, W. Challener, J. Hohlfeld, Y. Kubota, L. Li, B. Lu, C. Mihalcea, K. Mountfield, K. Pelhos, C. Peng, T. Rausch, M. Seigler, D. Weller, and X. Yang, "Heat-assisted magnetic recording," IEEE Trans. on Magn., vol. 42, no. 10, pp. 2417 -2421, Oct. 2006.

[2] J.-G. Zhu, X. Zhu, and Y. Tang, "Microwave assisted magnetic recording," IEEE Trans. on Magn., vol. 44, no. 1, pp. 125 -131, jan. 2008.

[3] B. Terris, T. Thomson, and G. Hu, "Patterned media for future magnetic data storage," Microsystem Technologies, vol. 13, pp. 189-196, 2007, 10.1007/s00542-006-0144-9. [Online]. Available: http://dx.doi.org/10.1007/s00542-006-0144-9

[4] R. Wood, M. Williams, A. Kavcic, and J. Miles, "The feasibility of magnetic recording at 10 terabits per square inch on conventional media," IEEE Trans. on Magn., vol. 45, no. 2, pp. 917 -923, Feb. 2009.

[5] J. F. Heanue, K. Gürkan, and L. Hesselink, "Signal detection for page-accessoptical memories with intersymbol interference," Appl. Opt., vol. 35, no. 14, pp. 2431-2438, May 1996. [Online]. Available: http://ao.osa.org/abstract.cfm?URI=ao-35-14-2431

[6] B. G. Roh, S. U. Lee, J. Moon, and Y. Chen, "Single-head/single-track detection in interfering tracks," IEEE Trans. on Magn., vol. 38, no. 4, pp. $1830-1838$, jul 2002

[7] A. Krishnan, R. Radhakrishnan, B. Vasic, A. Kavcic, W. Ryan, and F. Erden, "2-d magnetic recording: Read channel modeling and detection," IEEE Trans. on Magn., vol. 45, no. 10, pp. 3830 -3836, Oct. 2009.

[8] A. Kavcic, X. Huang, B. Vasic, W. Ryan, and M. Erden, "Channel modeling and capacity bounds for two-dimensional magnetic recording," Magnetics, IEEE Transactions on, vol. 46, no. 3, pp. 812 -818, march 2010.

[9] M. Khatami and B. Vasic, "Generalized belief propagation detector for tdmr microcell model," In preparation for Joint Magn. Magn. Mat. (MMM) IEEE Int. Magn. Conf. (Intermag), 2013.
[10] C. Modlin, K. Fisher, and J. Cioffi, "Analysis of and detector comparisons using the microtrack model of magnetic recording," IEEE Trans. on Magn., vol. 34, no. 1, pp. 63 -68, Jan. 1998.

[11] L. Bahl, J. Cocke, F. Jelinek, and J. Raviv, "Optimal decoding of linear codes for minimizing symbol error rate (corresp.)," IEEE Trans. on Inf. Theory, vol. 20, no. 2, pp. 284 - 287, mar 1974.

[12] B. M. Kurkoski, "Towards efficient detection of two-dimensional intersymbol interference channels," IEICE Trans. Fundamentals, vol. E91-A, no. 10 , Oct. 2008.

[13] J. Yedidia, W. Freeman, and Y. Weiss, "Constructing free-energy approximations and generalized belief propagation algorithms," IEEE Trans. on Inf. Theory, vol. 51, no. 7, pp. 2282 - 2312, Jul. 2005.

[14] O. Shental, N. Shental, S. Shamai (Shitz), I. Kanter, A. Weiss, and Y. Weiss, "Discrete-input two-dimensional gaussian channels with memory: Estimation and information rates via graphical models and statistical mechanics," IEEE Trans. on Inf. Theory, vol. 54, no. 4, pp. 1500 -1513, Apr. 2008.

[15] D. Wilton, D. McKirdy, H. Shute, J. Miles, and D. Mapps, "Approximate three-dimensional head fields for perpendicular magnetic recording," IEEE Trans. on Magn., vol. 40, no. 1, pp. 148 - 156, Jan. 2004.

[16] S. Nabavi and B. Vijaya Kumar, "Two-dimensional generalized partial response equalizer for bit-patterned media," in IEEE Int. Conf. on Comm., 2007. ICC '07., Jun. 2007, pp. 6249 -6254.

[17] S. Karakulak, P. Siegel, J. Wolf, and H. Bertram, "Joint-track equalization and detection for bit patterned media recording," IEEE Trans. on Magn., vol. 46, no. 9, pp. 3639 -3647, Sep. 2010.

[18] J. Kim and J. Lee, "Iterative two-dimensional soft output viterbi algorithm for patterned media," IEEE Trans. on Magn., vol. 47, no. 3, pp. $594-597$, Mar. 2011.

[19] X. Chen and K. Chugg, "Near-optimal data detection for twodimensional isi/awgn channels using concatenated modeling and iterative algorithms," in Communications, 1998. ICC 98. Conference Record. 1998 IEEE International Conference on, vol. 2, jun 1998, pp. $952-956$ vol.2.

[20] M. Marrow and J. Wolf, "Iterative detection of 2-dimensional isi channels," in Inf. Theory Workshop, 2003. Proc. 2003 IEEE, march-4 april 2003, pp. $131-134$.

[21] Y. Wu, J. O'Sullivan, N. Singla, and R. Indeck, "Iterative detection and decoding for separable two-dimensional intersymbol interference," IEEE Trans. on Magn., vol. 39, no. 4, pp. 2115 - 2120, July 2003.

[22] Y. Chen, P. Njeim, T. Cheng, B. Belzer, and K. Sivakumar, "Iterative soft decision feedback zig-zag equalizer for $2 \mathrm{~d}$ intersymbol interference channels," IEEE Jour. on Selected Areas in Communications, vol. 28, no. 2, pp. $167-180$, february 2010.

[23] Y. Chen and S. G. Srinivasa, "Signal detection algorithms for twodimensional intersymbol-interference channels," in IEEE International Symposium on Information Theory Proceedings (ISIT), Jul 2012. 